Check for updates

Cite this: RSC Adv., 2017, 7, 37436

Received 14th June 2017 Accepted 23rd July 2017

DOI: $10.1039 / \mathrm{c} 7 \mathrm{ra06647c}$

rsc.li/rsc-advances

\section{Crystallinity depends on choice of iron salt precursor in the continuous hydrothermal synthesis of $\mathrm{Fe}-\mathrm{Co}$ oxide nanoparticles}

\author{
Jian Liu, (D) a Isabella Römer, (DD ${ }^{b}$ Selina Vi Yu Tang, ${ }^{c}$ Eugenia Valsami-Jones ${ }^{b}$ \\ and Richard E. Palmer*d
}

\begin{abstract}
A series of Fe-Co oxide nanoparticles (NPs) were prepared by a continuous hydrothermal method using iron nitrate and ammonium iron citrate as alternative iron precursors. The crystallinity, Fe/Co composition and element spatial distribution in the synthesised NPs were investigated using X-ray diffraction (XRD), aberration-corrected scanning transmission electron microscopy (ac-STEM) imaging, energy-dispersive X-ray spectroscopy (EDX) and electron energy loss spectroscopy (EELS). Strong dependence on the choice of iron salt was observed. We demonstrate that the presence of ammonium citrate markedly improves the crystallinity of the NPs; an ordered cobalt ferrite alloy is formed. We suggest this is due to the formation of a homogenous reaction environment during the thermal decomposition of ammonium citrate, and the formation of complexes among citrate, Fe and Co ions.
\end{abstract}

\section{Introduction}

Magnetic nanoparticles (NPs) have shown great potential for a number of important technological applications, such as information storage, medical diagnosis, drug delivery, etc. ${ }^{1-3}$ For example, in an alternating magnetic field, magnetic NPs are able to convert electromagnetic energy into thermal energy, which is useful for treatment of cancer by magnetic hyperthermia., ${ }^{4,5} \mathrm{Fe}-\mathrm{Co}$ oxide NPs have demonstrated great potential linked to their distinctive properties. They show large magnetic anisotropy, moderate saturation magnetization, chemical stability and mechanical hardness. ${ }^{6-8}$ The magnetic properties of Fe-Co oxide NPs are believed to be related to their morphology, composition, and cation arrangements. ${ }^{\text {9-11 }}$ Joshi et $a l .{ }^{9}$ reported that the saturation magnetization of cobalt ferrite NPs increases with an increase in size, and that spherical NPs exhibit higher saturation magnetization than faceted irregular ones. The effect of stoichiometry, i.e. metal ratio, on the magnetic properties of cobalt ferrite NPs was studied by Sathya et al. ${ }^{\mathbf{1 0}}$ They reported that the specific absorption rate (relevant for magnetic hyperthermia) and the relaxivity value (significant for magnetic resonance imaging, MRI) of the cobalt ferrite $\left(\mathrm{Co}_{x} \mathrm{Fe}_{3-x} \mathrm{O}_{4}\right)$ NPs could be tuned not only by the NP's

\footnotetext{
${ }^{a}$ Nanoscale Physics Research Laboratory, School of Physics and Astronomy, University of Birmingham, Edgbaston, Birmingham B15 2TT, UK

${ }^{b}$ School of Geography, Earth and Environmental Sciences, University of Birmingham, Edgbaston, Birmingham, B15 2TT, UK

'Promethean Particles Ltd., 1-3 Genesis Park, Midland Way, Nottingham NG7 3EF, UK ${ }^{d}$ College of Engineering, Swansea University, Bay Campus, Fabian Way, Swansea, SA1 8EN, UK. E-mail: r.e.palmer@swansea.ac.uk
}

size, but also by the Fe/Co ratio. They concluded that particles of $20 \pm 2 \mathrm{~nm}$ in size and Co stoichiometry $(x)$ in the $x=0.5-0.7$ range are promising candidates as heat mediators for both hyperthermia and MRI applications. Fe-Co oxide NPs usually have a spinel structure with unit formula $\mathrm{AB}_{2} \mathrm{O}_{4}$, where $\mathrm{A}$ and $\mathrm{B}$ stand for tetrahedral and octahedral cation sites within the close packed array of oxygen atoms. ${ }^{12}$ The metal ion arrangement at different sites also has a strong influence on magnetic properties. Previous studies ${ }^{\mathbf{1 3 - 1 5}}$ have reported the valence states of the Fe and Co cations and their corresponding distributions in the A and B sites of the cobalt ferrite structure with Mössbauer spectra. Liu et al. ${ }^{11}$ reported that the migration of $\mathrm{Co}^{2+}$ ions from A to B sites could result in an increase in the saturation magnetization and coercivity.

The hydrothermal method is one of the most widely used synthesis protocols for the preparation of metal oxide NPs. ${ }^{\mathbf{1 6 , 1 7}}$ By adjusting the synthesis conditions, such as $\mathrm{pH}$, reaction temperature, Co to $\mathrm{Fe}$ ratio and surfactants, the particle morphology and Co to Fe ratio in the NPs can be adjusted. ${ }^{\mathbf{1 6 , 1 8 , 1 9}}$ The cation distribution in A or B sites can be tuned by changing the reaction time ${ }^{\mathbf{1 1}}$ or the quenching speed after post-annealing of the Fe-Co oxide NPs. ${ }^{12,20}$ However, the effect of synthesis conditions on the element spatial distribution of Co and Fe in the NPs is rarely discussed. The degree of inhomogeneous alloying in the NPs will undoubtedly affect their magnetic properties; Antoniak et al. ${ }^{21}$ found that the magnetic moment of FePt NPs was reduced by $20-30 \%$ with respect to the bulk material due to compositional inhomogeneity in the particles. Furthermore, López-Ortega et al. ${ }^{22}$ synthesized magnetic core/ shell NPs of $\mathrm{Mn}_{x} \mathrm{Fe}_{3-x} \mathrm{O}_{4} / \mathrm{Fe}_{x} \mathrm{Mn}_{3-x} \mathrm{O}_{4}$ using a seeded growth procedure; the heterostructured NPs showed a strong exchange 
coupling between the core and shell resulting in a coercivity increase.

Ac-STEM is a powerful tool for characterising binary complex NPs using Z-contrast imaging ${ }^{23,24}$ and STEM-EELS analysis. ${ }^{25-27}$ Here we studied the morphology, Fe/Co ratio and $\mathrm{Fe}$ and Co distribution of the hydrothermally prepared Fe-Co oxide NPs by ac-STEM. We found that by changing the iron salt, from iron nitrate to ammonium iron citrate, the internal order (crystallinity, elemental distribution, etc.) in the Fe-Co oxide NPs can be changed.

\section{Synthesis procedure and experimental method}

The synthesis of a series of Fe-Co oxide samples at Promethean Particles Ltd (Nottingham, UK) was conducted in water using a continuous-flow hydrothermal reactor, which has previously been described in the literature; ${ }^{28-32}$ a schematic diagram is shown in Fig. 1. Briefly, deionised water was pumped into an electric preheater at a rate of $20 \mathrm{ml} \mathrm{min}^{-1}$. The water was heated to $400{ }^{\circ} \mathrm{C}$ and flowed into the nozzle reactor section of the system, where it met a flow of aqueous metal salt solution at ambient temperature, pumped in at a rate of $10 \mathrm{ml} \mathrm{min}{ }^{-1}$.

For each sample, the metal salt solution was composed of the specified concentration ratio of iron and cobalt ions, with total concentration $0.05 \mathrm{M}$. The cobalt salt used was cobalt(II) acetate tetrahydrate $\left(\mathrm{Co}\left(\mathrm{C}_{2} \mathrm{H}_{3} \mathrm{O}_{2}\right)_{2} \cdot 4 \mathrm{H}_{2} \mathrm{O}\right.$, Sigma-Aldrich). Two different iron salts, iron(III) nitrate nonahydrate $\left(\mathrm{Fe}\left(\mathrm{NO}_{3}\right)_{3}\right.$ - $9 \mathrm{H}_{2} \mathrm{O}$, Sigma Aldrich) and ammonium iron(III) citrate $\left(\mathrm{C}_{6} \mathrm{H}_{8}\right.$ $\mathrm{O}_{7} \cdot x \mathrm{Fe}^{3+} \cdot y \mathrm{NH}_{3}$, Sigma-Aldrich), were used to synthesise sample series INN (iron nitrate nonahydrate) and AIC (ammonium iron citrate), respectively. A summary of the precursor concentrations used is given in Table 1.

For the AIC-1 sample, the water flow feed also contained $0.25 \% \mathrm{v} / \mathrm{v}$ hydrogen peroxide $\left(\mathrm{H}_{2} \mathrm{O}_{2}\right.$, Fisher Scientific, UK). Hydrogen peroxide was used here to drive oxidation of the cobalt precursor. A reaction temperature of $400{ }^{\circ} \mathrm{C}$ was chosen to keep a high rate of conversion and the whole system pressure was maintained at 240 bar by a backpressure regulator. The

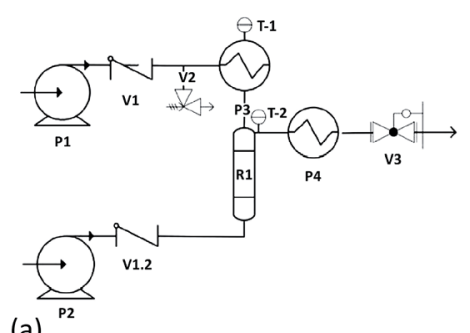

(a) (b)
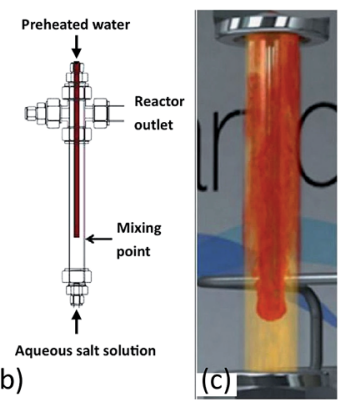

Fig. 1 (a) Flow diagram of continuous flow supercritical water reactor system: P1 and P2 - Gilson HPLC pumps, P3 - water preheater, R1 nozzle reactor, P4 - cooler, V1, V1.2 - check valves, V2 - pressure relief valve, V3 - Tescom backpressure regulator, T-1,2 - thermocouples. (b) Nozzle reactor design. ${ }^{29}$ (c) An animated depiction of the nozzle reactor. ${ }^{30}$
Table 1 Summary of precursor concentrations used for each sample

\begin{tabular}{lllll}
\hline $\begin{array}{l}\text { Sample } \\
\text { code }\end{array}$ & $\begin{array}{l}\text { Fe/Co } \\
\text { ratio }\end{array}$ & $\begin{array}{l}\text { Cobalt acetate } \\
(\mathrm{M})\end{array}$ & $\begin{array}{l}\text { Ammonium iron } \\
\text { citrate }(\mathrm{M})\end{array}$ & $\begin{array}{l}\text { Iron nitrate } \\
(\mathrm{M})\end{array}$ \\
\hline INN-1 & $1: 3$ & 0.0375 & - & 0.0125 \\
INN-2 & $1: 1$ & 0.0250 & - & 0.0250 \\
INN-3 & $3: 1$ & 0.0125 & - & 0.0375 \\
AIC-1 & $1: 3$ & 0.0375 & 0.0125 & - \\
AIC-2 & $1: 1$ & 0.0250 & 0.0250 & - \\
AIC-3 & $3: 1$ & 0.0125 & 0.0375 & -
\end{tabular}

collected samples were washed by centrifugation or filtration and then sent to the University of Birmingham for characterisation.

The samples were imaged in a $200 \mathrm{kV}$ JEM2100F (JEOL) STEM with a spherical aberration probe corrector (CEOS). Highangle annular dark field (HAADF) images were acquired with inner and outer detector semi-angles of 62 and $164 \mathrm{mrad}$ (camera length $10 \mathrm{~cm}$ ), and probe convergence semi-angle of 19 mrad. The elemental composition was determined by EDX spectra, which were obtained with a Bruker XFlash 4030 detector attached to the microscope. The EELS spectra were recorded with a Gatan Enfina detector at a camera length of $2 \mathrm{~cm}$ and a collection semi-angle of $57.8 \mathrm{mrad}$. Zeta potential measurements were made with a Malvern Zetasizer ZS system. Measurements were performed at $21{ }^{\circ} \mathrm{C}$ and repeated at least 5 times per sample using a low volume zeta potential cell, which was washed with ultra-high purity (UHP) water in between each sample. The X-ray diffraction (XRD) patterns were collected using a Bruker D8 powder diffractometer using $\mathrm{Cu} \mathrm{K} \alpha$ radiation $(\lambda=1.5406 \AA)$.

\section{Results and discussion}

Fig. 2 shows the HAADF images and corresponding histograms of NP diameter for the samples from the INN and AIC batches. In both cases, the NPs were aggregated. However, the level of agglomeration of the NPs in the AIC sample series was higher than that in the INN series, which agrees with the zeta potential measurements. The average zeta potential (shown in Table 2) of the INN samples is larger than the AIC series, indicating a higher stability and lower level of agglomeration. From the size distribution histograms, it can be seen that the diameter ranged from around 5 to $30 \mathrm{~nm}$, and that the AIC sample series had more large particles ( $>15 \mathrm{~nm}$ ). Overall, the average diameters of the two batches of samples were close to each other. Changing the iron salt in the solution seems to have an effect on the dispersion of the hydrothermally synthesised Fe-Co NPs.

The XRD results from the INN and AIC sample series are shown in Fig. 3. The XRD spectra of the INN samples (Fig. 3a) suggested that the Fe-Co oxide NPs from INN-2 and -3 are not well crystallised, since the diffraction peaks were weak and broad. The crystallite sizes of Fe-Co nanoparticles in the INN series are calculated using the Scherer equation, ${ }^{33}$

$$
D=K \lambda / \beta \cos \theta,
$$



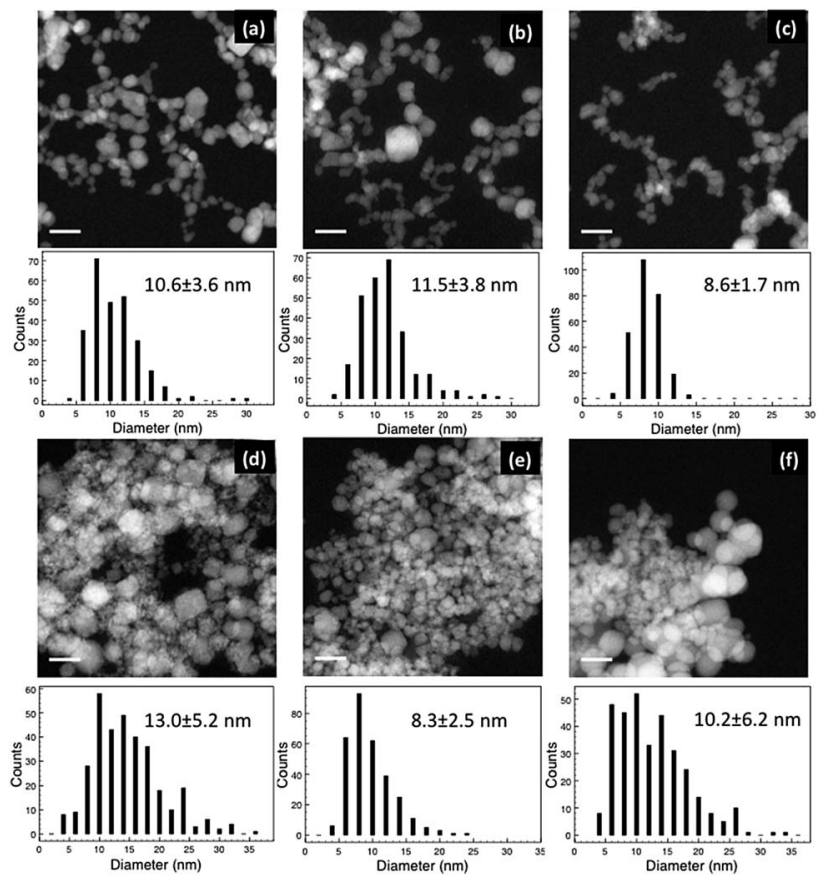

Fig. 2 HAADF images of sample (a) INN-1, (b) INN-2, (c) INN-3, (d) AIC-1, (e) AIC-2 and (f) AIC-3. The corresponding particle diameter distribution histograms are shown underneath. The scale bar is $20 \mathrm{~nm}$.

Table 2 Zeta potential measurements

\begin{tabular}{lc}
$\begin{array}{l}\text { Sample } \\
\text { code }\end{array}$ & Zeta potential $(\mathrm{mV})$ \\
\hline INN-1 & $17.8 \pm 0.6$ \\
INN-2 & $20.5 \pm 0.7$ \\
INN-3 & $19.7 \pm 0.5$ \\
AIC-1 & $18.8 \pm 0.4$ \\
AIC-2 & $8.5 \pm 0.3$ \\
AIC-3 & $-7.3 \pm 0.2$
\end{tabular}

where $D$ is the mean size of the crystalline domains, $K$ is the shape factor and takes the value 0.9 in the calculation, $\lambda$ is the $\mathrm{X}$ ray wavelength, $\beta$ is the full width half maximum (FWHM) of the (311) peak obtained from their Gaussian fittings, and $\theta$ is the Bragg angle. The average crystallite sizes for sample INN-1, -2 and -3 are $5.8 \mathrm{~nm}, 3.6 \mathrm{~nm}$ and $3.8 \mathrm{~nm}$, respectively. These values are much smaller than those measured from the STEM-HAADF images, which suggests that the Fe-Co NPs probably have segregated Fe-rich and Co-rich regions since these regions have different lattice parameters when the $\mathrm{Fe} / \mathrm{Co}$ ratio varies. ${ }^{15,34}$ This was also supported by the EELS mapping analysis shown below. Fig. 3 b shows clear XRD patterns from the AIC samples, which correspond to the $\mathrm{AB}_{2} \mathrm{O}_{4}$ structure. ${ }^{35,36}$ The average FWHM value of the (311) peaks for the AIC samples is $0.9^{\circ}$, which is much smaller than that of the INN series (about $2.0^{\circ}$ ). This indicates that these particles have bigger crystalline domains than those in the INN sample series. The average crystallite sizes calculated from XRD patterns of AIC-1, -2 and -3 are around $11.4 \mathrm{~nm}$, $7.9 \mathrm{~nm}$ and $9.1 \mathrm{~nm}$, respectively, which are very close to the
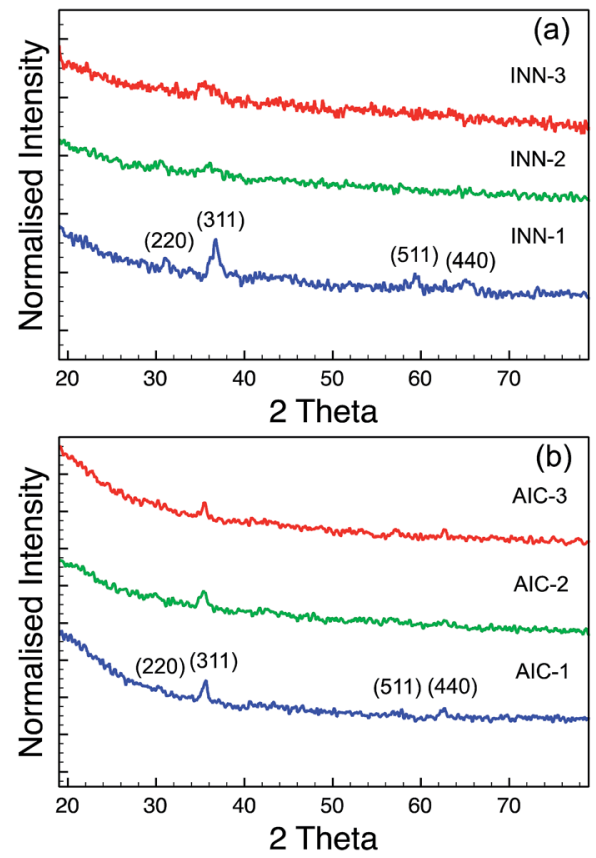

Fig. 3 XRD patterns of the sample series (a) INN and (b) AIC.

microscope measurements, indicating a high level of crystallinity in the particles.

Table 3 shows the elemental compositions of each sample in the INN and AIC series. It is important to note that the $\mathrm{Fe} / \mathrm{Co}$ ratios of the synthesised INN samples are close to the ratios in the metal salt solutions. In the case of the AIC samples, these two ratios are very different, and the $\mathrm{Fe} / \mathrm{Co}$ ratios detected in the particles are always higher than in the solutions. This may be caused by the ammonium citrate in the solutions. In the hydrothermal synthesis of iron oxide particles from ammonium iron citrate, it has been reported that the $\mathrm{Fe}^{3+}$ can be reduced to $\mathrm{Fe}^{2+}$ by the carbon monoxide formed from the thermal decomposition of ammonium citrate in the supercritical water. ${ }^{37,38}$ In a reducing environment, the oxidation of $\mathrm{Co}^{2+}$ to $\mathrm{Co}^{3+}$ will also be highly suppressed and the precipitation of Co ions from the solution will become more difficult. ${ }^{16}$ This could explain why the Co content detected in the AIC samples was always lower than expected. For the samples in the INN series, where iron nitrate was used as the precursor, the nitrate ions might act as an oxidation agent to drive the $\mathrm{Co}^{2+}$ to $\mathrm{Co}^{3+}$.

Table 3 Elemental compositions of the samples from INN and AIC batches determined by STEM-EDX

\begin{tabular}{llllr}
\hline & \multicolumn{4}{c}{ Element $(\mathrm{at} \%)$} \\
\cline { 3 - 5 } $\begin{array}{l}\text { Sample } \\
\text { code }\end{array}$ & Fe/Co ratio & \multicolumn{1}{l}{$\mathrm{O}$} & Fe & \multicolumn{1}{c}{ Co } \\
\hline INN-1 & $0.37 \pm 0.02$ & $62.2 \pm 2.4$ & $10.2 \pm 0.4$ & $27.6 \pm 1.0$ \\
INN-2 & $0.91 \pm 0.05$ & $61.0 \pm 2.7$ & $18.6 \pm 0.8$ & $20.5 \pm 0.8$ \\
INN-3 & $3.17 \pm 0.15$ & $66.4 \pm 2.4$ & $25.6 \pm 0.8$ & $8.1 \pm 0.3$ \\
AIC-1 & $1.87 \pm 0.08$ & $58.4 \pm 1.9$ & $27.1 \pm 0.8$ & $14.5 \pm 0.5$ \\
AIC-2 & $2.56 \pm 0.11$ & $62.6 \pm 1.9$ & $26.9 \pm 0.8$ & $10.5 \pm 0.3$ \\
AIC-3 & $3.22 \pm 0.12$ & $51.5 \pm 1.4$ & $37.0 \pm 1.0$ & $11.5 \pm 0.3$
\end{tabular}


The element spatial distribution of the metals in the samples was studied by STEM-EELS and some mapping results are shown in Fig. 4. We found that the element spatial distributions in the NPs synthesised with iron nitrate (INN batch) were not uniform, as is shown in Fig. 4a and b. A Co-rich region tended to lie at the surface of the particles and an Fe-rich area in the core. However, a complete core-shell structure was not observed, and in some cases, even a "dumbbell" distribution of Fe and Co was also observed. The non-uniform elemental distribution in the NPs of INN samples may be caused by the nitrate anions in the iron salt and the solubility of the Fe and Co ions in the solutions. Previous studies ${ }^{16,39}$ implied that Co oxide NPs could not be precipitated from cobalt nitrate solutions in the absence of a base, or that only a few large particles could be produced when Co nitrate was in contact with supercritical water. ${ }^{40}$ In contrast, the solubility of Fe ions in the iron nitrate solution is several orders of magnitude lower than that of Co in its nitrate solution. ${ }^{16}$ Therefore, the nucleation of $\mathrm{Fe}$ oxide or hydroxide particles is easier than that of Co oxide or hydroxide during hydrothermal processing. Consequently, the Co cations may then proceed to precipitate on the surface of Fe oxide particles due to ion diffusion in the solution, forming Co and Fe-rich areas. This also explains why the Fe-rich regions tend to be in the core and Co-rich regions on the surface.

For the AIC sample series, we found that the Fe and Co spatial distributions in these NPs were very uniform (shown in Fig. 4c and d). In this case, cobalt ferrite is the likely product as the XRD results indicate. There are two possible reasons for the uniform elemental distributions. First, the $\mathrm{CO}$ molecules produced by the

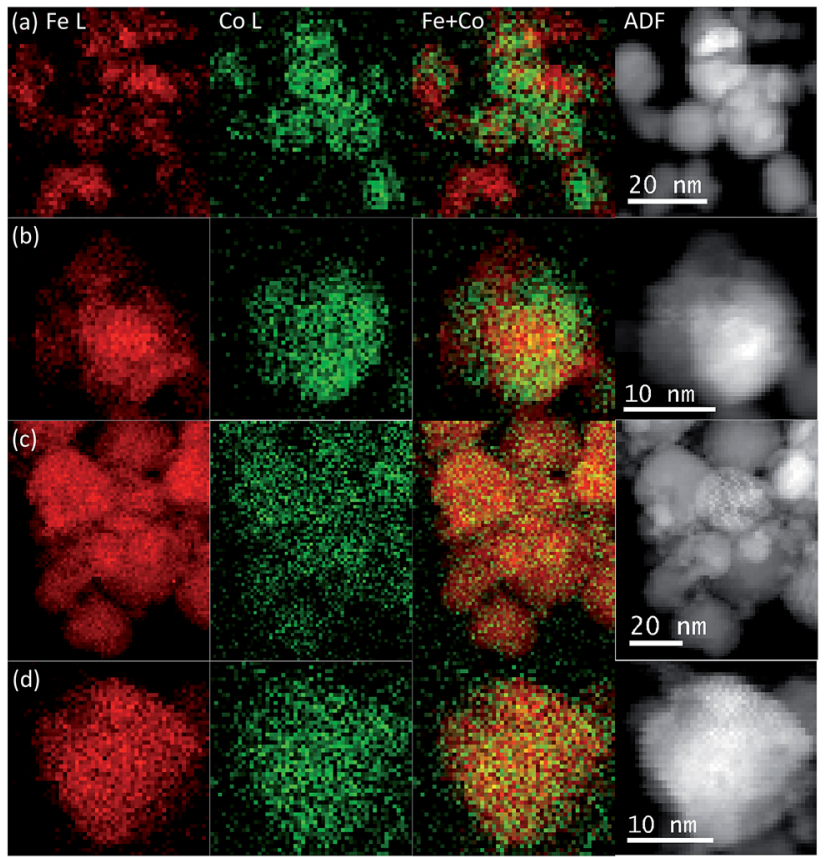

Fig. 4 Examples of EELS mapping of samples INN-2 (a) and AIC-2 (c) (b and d) Are single nanoparticle EELS maps from INN-2 and AIC-2, respectively. From left to the right we show: the $\mathrm{Fe}$ (red) and Co (green) $\mathrm{L}$ edge maps, a mix of the Fe and Co maps, and the corresponding ADF images. thermal decomposition of citrate ${ }^{37,41}$ are expected to be miscible with supercritical water and provide a homogeneous reaction environment promoting the oxidizing and reducing capability. ${ }^{38}$ Second, citrate anions can form complexes with Fe and Co cations, respectively. Citrate complexes of $\mathrm{Fe}$ or Co have been reported elsewhere in the literatures; ${ }^{42,43}$ the citrate ligand binds to $\mathrm{Fe}$ or Co atoms through the $\mathrm{O}$ atom of the hydroxyl group and the carboxyl group. A formation of these complexes (among Fe ions, Co ions and citrate) in the cobalt iron salts solution will promote the simultaneous precipitation of $\mathrm{Fe}$ and $\mathrm{Co}$ ions during the thermal decomposition process, ${ }^{41,44}$ resulting in a uniform elemental distribution.

Controlling the elemental arrangement in magnetic alloy NPs is of great importance, not only because it can change the magnetic properties of the particles ${ }^{21}$ but also because it might influence their toxicities and biocompatibilities. Iron oxide NPs are widely used in in vivo applications because of their low toxicity and biocompatibility. ${ }^{45,46}$ For the Fe-Co oxide NPs, the non-uniform distribution of Co oxide (especially when the Co rich region is on the surface of the NPs) may increase the toxicity of the particles, since it has been reported that Co NPs can induce cytotoxicity and genotoxicity in cells. ${ }^{4-49} \mathrm{~A}$ toxicity investigation of the Fe-Co oxide NPs reported in this work is in progress, and may shed light on the effect of the spatial distributions of the metals in the NPs on the toxicity.

\section{Conclusions}

In summary, we have explored a continuous hydrothermal method to prepare Fe-Co oxide NPs using two different iron salts as precursors. We found that the iron salt anions have a significant effect on the degree of crystallinity and aggregation, composition of the NPs, and spatial distribution of the metals in the NPs. The Fe-Co oxide NPs prepared with ammonium iron citrate were found to be well crystallised and had a more uniform Fe and Co distribution than the particles synthesised with an iron nitrate precursor. We suggest that ammonium citrate provides homogeneous reaction environment and promotes the simultaneous precipitation of Fe and Co ions by forming complexes. Overall, our investigation into the effect of precursor salt on the continuous hydrothermal synthesis is helpful to understanding the reaction process, allowing for better control of the NPs produced.

\section{Acknowledgements}

We thank Wolfgang Theis for a careful reading of this manuscript. We thank the EPSRC (EP/K006061/1) and the European Commission's 7th Framework Programme project "NanoMILE" (contract no. NMP4-LA-2013-310451) for financial support. The STEM instrument employed in this research was obtained through the Birmingham Science City project "Creating and Characterising Next Generation Advanced Materials," supported by Advantage West Midlands (AWM) and funded in part by the European Regional Development Fund (ERDF). JL acknowledges the Chinese Scholarship Council and the University of Birmingham for funding of his doctorate studies. 


\section{Notes and references}

1 A. K. Nikumbh, R. A. Pawar, D. V. Nighot, G. S. Gugale, M. D. Sangale, M. B. Khanvilkar and A. V. Nagawade, J. Magn. Magn. Mater., 2014, 355, 201-209.

2 S. Mornet, S. Vasseur, F. Grasset and E. Duguet, J. Mater. Chem., 2004, 14, 2161-2175.

3 P. K. Jain, X. Huang, I. H. El-Sayed and M. A. El-Sayed, Acc. Chem. Res., 2008, 41, 1578-1586.

4 J. Lee, J. Jang, J. Choi, S. H. Moon, S. Noh, J. Kim, J. G. Kim, I. Kim, K. I. Park and J. Cheon, Nat. Nanotechnol., 2011, 6, 418-422.

5 E. Fantechi, C. Innocenti, M. Zanardelli, M. Fittipaldi, E. Falvo, M. Carbo, V. Shullani, L. Di Cesare Mannelli, C. Ghelardini, A. M. Ferretti, A. Ponti, C. Sangregorio and P. Ceci, ACS Nano, 2014, 8, 4705-4719.

6 F. Tihay, G. Pourroy, M. Richard-Plouet, A. C. Roger and A. Kiennemann, Appl. Catal., A, 2001, 206, 29-42.

7 D. S. Mathew and R. S. Juang, Chem. Eng. J., 2007, 129, 51-65. 8 B. G. Toksha, S. E. Shirsath, S. M. Patange and K. M. Jadhav, Solid State Commun., 2008, 147, 479-483.

9 H. M. Joshi, Y. P. Lin, M. Aslam, P. V. Prasad, E. A. SchultzSikma, R. Edelman, T. Meade and V. P. Dravid, J. Phys. Chem. C, 2009, 113, 17761-17767.

10 A. Sathya, P. Guardia, R. Brescia, N. Silvestri, G. Pugliese, S. Nitti, L. Manna and T. Pellegrino, Chem. Mater., 2016, 28, 1769-1780.

11 M. Liu, M. Lu, L. Wang, S. Xu, J. Zhao and H. Li, J. Mater. Sci., 2016, 51, 5487-5492.

12 G. A. Sawatzky, J. Appl. Phys., 1968, 39, 1204.

13 S. Kawano, N. Yamamoto and N. Achiwa, Jpn. J. Appl. Phys., 1974, 13, 1891-1892.

14 P. J. Murray and J. W. Linnett, J. Phys. Chem. Solids, 1976, 37, 1041-1042.

15 P. A. Smith, C. D. Spencer and R. P. Stillwell, J. Phys. Chem. Solids, 1978, 39, 107-111.

16 L. J. Cote, A. S. Teja, A. P. Wilkinson and Z. J. Zhang, J. Mater. Res., 2003, 210, 307-317.

17 T. J. Daou, G. Pourroy, S. Begin-Colin, J. M. Greneche, C. Ulhaq-Bouillet, P. Legare, P. Bernhardt, C. Leuvrey and G. Rogez, Chem. Mater., 2006, 18, 4399-4404.

18 D. Zhao, X. Wu, H. Guan and E. Han, J. Supercrit. Fluids, 2007, 42, 226-233.

19 L. Zhao, H. Zhang, Y. Xing, S. Song, S. Yu, W. Shi, X. Guo, J. Yang, Y. Lei and F. Cao, J. Solid State Chem., 2008, 181, 245-252.

20 G. A. Sawatzky, F. Van Der Woude and A. H. Morrish, Phys. Rev., 1969, 187, 747-757.

21 C. Antoniak, M. Spasova, A. Trunova, K. Fauth, F. Wilhelm, A. Rogalev, J. Minár, H. Ebert, M. Farle and H. Wende, J. Phys.: Condens. Matter, 2009, 21, 336002.

22 A. López-Ortega, M. Estrader, G. Salazar-Alvarez, S. Estradé, I. V. Golosovsky, R. K. Dumas, D. J. Keavney, M. Vasilakaki, K. N. Trohidou, J. Sort, F. Peiró, S. Suriñach, M. D. Baró and J. Nogués, Nanoscale, 2012, 4, 5138-5147.
23 N. Jian and R. E. Palmer, J. Phys. Chem. C, 2015, 119, 1111411119.

24 F. Yin, Z. W. Wang and R. E. Palmer, J. Am. Chem. Soc., 2011, 133, 10325-10327.

25 I. Romer, Z. Wang, R. C. Merrifield, R. E. Palmer and J. R. Lead, Environ. Sci. Technol., 2016, 50, 2183-2190.

26 M. Baalousha, K. P. Arkill, I. Romer, R. E. Palmer and J. R. Lead, Sci. Total Environ., 2015, 502, 344-353.

27 Y. Li, Z. W. Wang, C.-Y. Chiu, L. Ruan, W. Yang, Y. Yang, R. E. Palmer and Y. Huang, Nanoscale, 2012, 4, 845-851.

28 H. Hobbs, S. Briddon and E. Lester, Green Chem., 2009, 11, 484-491.

29 E. Lester, G. Aksomaityte, J. Li, S. Gomez, J. GonzalezGonzalez and M. Poliakoff, Prog. Cryst. Growth Charact. Mater., 2012, 58, 3-13.

30 F. Moro, S. V. Y. Tang, F. Tuna and E. Lester, J. Magn. Magn. Mater., 2013, 348, 1-7.

31 E. Lester, S. V. Y. Tang, A. Khlobystov, V. L. Rose, L. Buttery and C. J. Roberts, CrystEngComm, 2013, 15, 3256.

32 Q. Wang, S. V. Y. Tang, E. Lester and D. O'Hare, Nanoscale, 2013, 5, 114-117.

33 P. Scherrer, Nachr. Ges. Wiss. Goettingen, Math.-Phys. Kl., Fachgruppe 2, 1918, 96.

34 T. A. S. Ferreira, J. C. Waerenborgh, M. H. R. M. Mendonça, M. R. Nunes and F. M. Costa, Solid State Sci., 2003, 5, 383-392.

35 Y. I. Kim, D. Kim and C. S. Lee, Phys. B, 2003, 337, 42-51.

36 Z. Ma, L. Ren, S. Xing, Y. Wu and Y. Gao, J. Phys. Chem. C, 2015, 119, 23068-23074.

37 T. Adschiri, K. Kanazawa and K. Arai, J. Am. Ceram. Soc., 1992, 75, 1019-1022.

38 T. Adschiri, Y. Hakuta and K. Arai, Ind. Eng. Chem. Res., 2000, 39, 4901-4907.

39 L. J. Cote, A. S. Teja, A. P. Wilkinson and Z. J. Zhang, J. Mater. Res., 2002, 19, 2410-2416.

40 Y. Hao and A. Teja, J. Mater. Res., 2003, 18, 415-422.

41 N. S. Gajbhiye and S. Prasad, Thermochim. Acta, 1996, 285, 325-336.

42 T. Birsa Čelič, Z. Jagličič, K. Lazar and N. Zabukovec Logar, Acta Crystallogr., 2013, 69, 490-495.

43 Z. Zhou, Y. Deng and H. Wan, Cryst. Growth Des., 2005, 5, 1109-1117.

44 S. Prasad, A. Vijayalakshmi and N. S. Gajbhiye, J. Therm. Anal. Calorim., 1998, 52, 595-607.

45 R. Weissleder, D. D. Stark, B. L. Engelstad, B. R. Bacon, C. C. Compton, D. L. White, P. Jacobs and J. Lewis, Am. J. Roentgenol., 1989, 152, 167-173.

46 Y. Wu, Y. Wang, G. Luo and Y. Dai, Bioresour. Technol., 2009, 100, 3459-3464.

47 E. Papis, F. Rossi, M. Raspanti, I. Dalle-Donne, G. Colombo, A. Milzani, G. Bernardini and R. Gornati, Toxicol. Lett., 2009, 189, 253-259.

48 J. Ponti, E. Sabbioni, B. Munaro, F. Broggi, P. Marmorato, F. Franchini, R. Colognato and F. Rossi, Mutagenesis, 2009, 24, 439-445.

49 S. Chattopadhyay, S. K. Dash, S. Tripathy, B. Das, D. Mandal, P. Pramanik and S. Roy, Chem.-Biol. Interact., 2015, 226, 58-71. 